\title{
Sobre un caso de duplicidad genital femenina
}

\author{
Doctor Hernando Navas Angel (*)
}

Trabajo leído en la sesión de la Sociedad Colombiana de Obstetricia y Ginecología el 14 de noviembre de 1957.

Para estudiar debidamente las malformaciones uterinas congénitas, lo primero ha de ser recordar algunas nociones de embriología; vendrá luego una suscinta clasificación anatómica de ellas y por último han de considerarse sus aspectos ginecológicos J obstétricos. Circunscribiendo nuestras anotaciones al caso que reportamos, haremos al lector gracia de aquello que en detalles de clasificación y aspectos clínicos no guarde relación con él.

Las trompas de Falopio, el útero y la vagina se originan embriológicamente en los canales de Müller. Estos canales, pares, simétricos y paralelos, comprenden esquemáticamente dos partes separadas por el cruzamiento del "gubernaculum", que ha de constituir el ligamento redondo (1). La porción de cada canal de Müller situada por encima del "gubernaculum" permanece independiente y constituirá más tarde la trompa de Falopio. La porción inferior, en cambio, se suelda con la porción análoga del ca_ nal del otro lado, produciéndose normalmente la desaparición completa del tabique formado por esta fusión. De este proceso evolutivo resulta un único canal útero vaginal en el que poco a poco se identificarán útero y vagina.

El proceso evolutivo embriológico puede sufrir anomalías de diversos grados (1):

10 Si los canales de Müller permanecen independientes o se sueldan incompletamente, se constituirá la duplicidad uterina, más o menos extendida según el mayor o menor grado de soldadura de los canales.

(*) El autor: Miembro de Número de la Sociedad Colombiana de Obstetricia y Ginecología. Jefe de Clínica Obstétrica por Concurso (Universidad Nacional). 1953-54; 1955-56. 
20 Si el tabique formado por la unión de los dos canales per. siste, o desaparece solo en parte, resultará de ello el útero tabicado.

3 o Si uno de los dos canales detiene su evolución y se atrofia, en tanto que el otro evoluciona solo, se formará el útero unicórneo.

La etiopatogenia de las malformaciones genitales (2) es prácticamente desconocida a pesar de las variadísimas teorías que se han expuesto para explicarlas, entre las que podemos enumerar: brevedad de los ligamentos redondos, bridas de la alantoides, peritonitis fetal, alejamiento entre s- de los canales de Müller, interposición entre ellos de otros órganos, interposición de restos wolfianos, existencia de tumores, factores herenciales, factores atávicos, factores raciales, alcoholismo, sífilis, etc. Pero de todas estas posibles causas ninguna es aplicable a todos los casos y el problema etiopatogénico de las malformaciones genitales sigue sin solución.

En 1906 Ombredanne y Martín hicieron una clasificación de las malformaciones útero-vaginales que por su gran valor prác. tico es la preferida actualmente en la mayoría de las escuelas obstétricas. Dicha clasificación explica y aclara el mecanismo de cada malformación, relacionándolo con la época del desarrollo clel embrión en que se produjo la detención o la desviación de todo o parte del órgano afectado (2).

Las malformaciones uterinas pueden agruparse en tres ca. tegorías, a saber (1):

a) Uteros dobles.

b) Uteros tabicados.

c) Otras malformaciones.

a) En el grupo de los úteros dobles se encuentran:

1) Utero didelfo, producto de detención precoz del desarro1lo, en que el defecto de fusión comprometió toda la longitud del canal, dejando como resultado dos cuerpos uterinos distintos, dos cuellos separados y una vagina tabicada en toda su longitud.

2) Utero pseudo-didelfo, resultado de una detención de desarrollo cuando los canales de Müller iniciaban su fusión, haciendo esta incompleta y comprometiendo en la anomalía la porción inferior del órgano. Resultan de este fenómeno dos cuerpos uterinos totalmente separados, dos cuellos unidos y una vagina tabicada o única. 
3) Utero bicorne unicervical, que representa un grado más avanzado en el desarrollo embriológico. La soldadura se efectúa en casi toda la longitud de los canales, habiendo bifidez solamente en la porción más superior. Habrá, pues, una sola vagina, un solo cuello y una sola cavidad uterina en la parte inferior del órgano, pero en la parte superior de éste tendremos dos cuernos uterinos muy diferenciados, iguales o desiguales en su desarrollo. El caso que más adelante reportamos quedar a clasificado en el subgrupo de los úteros didelfos.

En relación con los úteros dobles, y además de las anteriores características anatómicas, hay otras de interés quirúrgico que no pueden ser dejadas sin mención. En los cuerpos uterinos del útero doble pueden verse diversas modalidades: pueden ser semejantes, simétricos, del mismo tamaño y forma o bien, completamente desiguales; uno de los dos pueden tener desarrollo normal, siendo el otro pequeño y deformado. En el útero bicorne particularmente, los dos cuernos pueden ser notoriamente diferen. tes, siendo a veces uno de ellos tan atrófico que simule una especie de apéndice implantado sobre un útero normal.

Los cuerpos uterinos, especialmente en el didelfo verdadero, forman entre s, por su inclinación lateral, un ángulo de abertura y orientación variable. Cuando uno de los dos está fuerte. mente apartado de la línea media hacia su lado respectivo, puede hacer muy peligrosa la histerectomía para el uréter correspondiente, sobre el cual se coloca.

Entre los dos cuerpos uterinos separados puede encontrarse un tabique peritoneal antero-posterior, extendido desde la vejiga hasta el cólon pélvico o el recto. Este tabique, blanco nacarado, es poco o nada vascularizado; atrae hacia atrás la vejiga y hacia adelante el cólon por lo que su sección quirúrgica debe hacerse cuidadosamente para no herir divertículos de estos órganos, posiblemente existentes en su espesor.

Los vasos uterinos, a pesar de la duplicidad del órgano, son normales en trayecto y número. Así, pues, el borde interno de cada hemi-útero es siempre avascular.

b) En el grupo de los úteros tabicados, a diferencia de los cobles en que la anomalía se advierte a primera vista exterior del órgano, se aprecia exteriormente una conformación más o menos normal. En ellos la anomalía es interna y solo el corte del órgano la hace aparente', siendo ella el resultado de la incompleta desaparición del tabique formado al fusionarse los dos cana. 
les de Müller. La persistencia de él divide el útero en dos cavidades, total o parcialmente separadas. Los úteros tabicados se subdividen en:

1) Utero bilocular total, o "bipartitus" con persistencia completa del tabique; 2) Utero bilocular corporal, o "subseptus", en que el tabique solo persiste en el fondo de la cavidad, formando un espolón antero_posterior, y 3)Utero bilocular cervical, o "infraseptus", en que el tabique persiste en la parte inferior divi. diendo el cuello en dos orificios que desembocan en una cavidad uterina única.

c) Entre las otras malformaciones son de enumerar:

1) El útero unicorne, excepcional y de origen embriológico ya visto; es largo, estrecho, con un solo cuerno y por ende con una sola trompa. 2) El útero cordiforme, que en su anomalía está muy cerca del órgano normal. Se caracteriza por una depresión en el fondo uterino que corresponde a una saliente mediana y redondeada en la cavidad del órgano, y 3) El útero hipoplásico. o infantil, acompañado en su deficiente desarrollo por todos los demás elementos de la esfera genital, incluyendo también a veces el esqueleto pélvico.

Como el repliegue urogenital da origen al canal de Müller y así mismo al ovario, al riñón y al uréter, sucede frecuentemente que las anomalías útero-vaginales son concomitantes con deformidades o ausencia de un ovario, riñón o uréter, o con desplaza. mientos orgánicos como el "riñón pélvico" (6), (3), (4). Undergerliter y Woolf, citados por Echeverri, se refieren a la coexistencia de deformaciones esqueléticas a nivel de las vértebras lumbares y sacras, de espina bífida y además de deformaciones de la porción terminal del tubo digestivo (6).

Diversas son las causas que llevan a la paciente con malformación uterina, casi siempre sin gravidez, a la consulta ginecológica: dismenorrea, dispareunia, esterilidad, aborto, y es enton. ces cuando puede descubrirse la anomalía útero-vaginal. La comprobación de la existencia de tabiques o diafragmas vaginales no tiene dificultad alguna, pero en cambio el simple examen ginecológico, orientado por el hallazgo hecho en la vagina, no permi. te diagnosticar la malformación uterina y menos aún cuando el cervix es único; se apela entonces al examen radiológico que po. ne en claro el diagnóstico. Como es natural, el estado grávido aumenta las dificultades para diagnosticar las malformaciones genitales: si la preñez es reciente, el cuerno vacío de un útero di- 
delfo, o el cuerpo no grávido de uno doble, pueden confundirse con un mioma y el coexisten dolor y hemorragia, puede presu. mirse el embarazo ectópico (1), (5).

Cuando la preñez localizada en uno de los cuerpos del útero doble ha llegado a la segunda mitad, la marcada lateralidad del grávido y el encuentro de un solo ligamento redondo, tenso y fuerte', inducirán al diagnóstico de la malformación de duplicidad, lo que se corrobora con el tacto vaginal cuando éste descubre la presencia de dos cuellos o una vagina tabicada. Las posibilidades de diagnóstico pueden verse aplazadas en el embarazo a término cuando la presentación está encajada, hasta cuando llega el momento de la extracción manual de placenta, de frecuente necesidad en estos casos, en cuya ejecución se puede des. cubrir fácilmente la duplicidad orgánica.

Con útero doble la menstruación es a menudo normal aunque puede ser hiper y poli-menorreica, con dos pérdidas en cada mes, en que una regla normal alterna con otra menos copiosa y de diferente aspecto, dando lugar a pensar en la hematometría, de causa difícil de establecer si se desconoce la malformación (1). Los dos cuerpos o cuernos uterinos pueden menstruar simultáneamente o hacerlo en forma alternativa, uno en cada mes (3).

La fecundidad de la mujer afectada de malformación uteri. na depende del grado e importancia de la anomalía. La imperfo. ración del himen o defectos vaginales como tabiques, bridas o diafragmas, pueden ser fácilmente corregidos por la cirugía (2). Cuando la duplicidad afecta la vagina, el coito no presenta dificultades porque se efectúa utilizando una u otra hemivagina, o la de mayor tamaño cuando las dos no son iguales (3).

Sin embargo, la malformación puede acompañarse de anomalías de las trompas y especialmente de hipofuncionalismo ovárico y entonces la esterilidad podría ser definitiva (2). Fuera de estos casos y de los de hipoplasia acentuada, las mujeres portadoras de úteros dobles son por lo general tan fecundas como las demás y aun a veces pueden llevar su fertilidad hasta hacer embarazos dobles, simultáneos o en tiempos distintos, en sus dos cavidades uterinas. En este último caso el embarazo es doble por el mecanismo de superfetación (1).

También, y éste es el caso de nuestra paciente, los embarazos pueden ser únicos y alternar sucesivamente en uno y otro de los dos úteros, cuando ambos tienen igual valor funcional y comunicación con el exterior. 
Producido el embarazo importa saber cómo puede compor. tarse cada uno de los dos úteros: a) el no grávido y b) el grávido. a) Lo más frecuente es la sinergia funcional absoluta con amenorrea en los dos lados y participación por parte del útero vacío de las modificaciones anatómicas sufridas por su congénere (1): aumento de volumen, reblandecimiento y modificaciones histológicas de la mucosa que van a constituir una caduca (1). Sin embargo la sinergia funcional puede no existir y a pesar de la gravidez de uno, el otro útero presenta hemorragias mens. truales. b) Con frecuencia el embarazo evoluciona normalmente hasta llegar a término, sobre todo si el útero favorecido es el me. jor constituido (1); esto ocurre, según Werth, citado por Nubiola, en el $26 \%$ de los casos. Pero surgen a veces accidentes más o menos graves, ya que este tipo de úteros es de musculatura deficiente. La asimetría de forma es causa de dificultades de interpretación y la confusión con un tumor uterino o con un embarazo ectópico, pueden llevar a una intervención quirúrgica inútil. La prudentie expectativa, en cambio, es susceptible de permitir el reconocimiento de la verdadera anomalía (5), aunque no debe olvidarse que esta expectativa solo puede adoptarse manteniendo a la paciente en medio hospitalario desde el momento en que se haga el diagnóstico de gravidez con duplicidad uterina, porque debido a la deficiencia muscular de los órganos uterinos, la distensión puede provocar alrededor del $44^{\circ}$ o $5^{\circ}$ mes la ruptura uterina con el dramático cuadro de hemorragia interna $\mathbf{y}$ shock peritoneal (2). En tal caso, solo la laparotomía de urgencia y la transfusión de sangre total pueden salvar la vida de la paciente. La frecuencia de este accidente en gravidez con útero do_ ble, según Werth, es de $45 \%$ y según Fehr, de $75 \%$.

Fuera de las eventualidades anteriores y también determinado por la deficiencia muscular uterina, puede producirse el aborto que a veces alterna en la misma mujer con partos a término, según cual sea el útero grávido en cada ocasión y su mayor o menor capacidad funcional.

Por extraño que parezca, cabe esperar la evolución normal del parto en los úteros malformados, pero ésta no es la más frecuente' ocurrencia (1). El útero vacío participa durante el traba. jo de las modificaciones fisiológicas que sufre el grávido. En efec. to, presenta también contracciones típicas, isócronas con las del útero gestante, su cuello se borra y también se dilata. 
Los accidentes más frecuentes son los trastornos que afectan a la fisiología del trabajo, a causa de la mala musculatura uterina (1), (2). El útero presenta contracciones débiles, irregulares y espaciadas; la formación del segmento, la incorporación, el borramiento y la dilatación del cuello se verifican muy lentamente, haciendo el trabajo peligrosamente largo. A esta distocia dinámica de hiposistolia se agregan las anomalías histológicas del cuello, todo lo cual contribuye con frecuencia a no dejar practicable ninguna conducta distinta de la operación cesárea.

Es también de temer la ruptura uterina durante el trabajo, así mismo favorecida por la deficiencia de la pared uterina.

Pero el más frecuente de los problemas que se presentan en el parto con útero doble, es la distocia mecánica creada por el útero vacío, que puede enclavarse en la pelvis desempeñando el papel de un tumor previo, como sucedió en el caso que más adelante reportamos. Ante esta emergencia es fácil la confusión con un mioma pediculado (2), cuando la duplicidad genital no alcanza al cuello y a la vagina. Sobra decir que ante la presencia ae un tumor previo, con o sin diagnóstico de su naturaleza, la conducta debe ser la práctica de la operación cesárea.

El alumbramiento por lo regular se verifica sin accidentes, aunque el desprendimiento placentario puede retardarse debido a la insuficiencia muscular o a la inercia secundaria determinada por el trabajo prolongado. Estos dos factores son también determinantes de la hemorragia post-alumbramiento.

El puerperio puede verse complicado por involución lenta del útero, por retención de loquios y por infección consecutiva. Alrededor del 4o o $5^{\circ}$ día se realiza la expulsión de la caduca del útero no grávido y de su retención resulta el problema séptico ute_ rino por desprendimiento y retención.

En cuanto a la frecuencia de las malformaciones uterinas por trastorno en la fusión de los canales de Müller, Echeverri cita a Eiseman y a Woolf, de los cuales el primero dice que se presenta un caso por 1.500 de obstetricia y uno por 2.000 de ginecología; el segundo afirma que esta proporción debe ser mayor ya que muchos casos pasan inadvertidos y otros no son reportados (6).

Desde el punto de vista del tratamiento solamente tienen interés los úteros dobles (1). Ya habíamos anotado que una vez producido el embarazo, la expectativa es la única conducta lógica, a menos que su evolución presente trastornos serios, no olvi- 
dando la frecuencia del aborto y más especialmente la frecuencia de la retención de éste. Iniciado el trabajo de parto, la paciente, hospitalizada desde el momento en que se hizo el diagnóstico de gravidez, debe ser objeto de cuidadosa vigilancia. Si las condiciones del trabajo permiten esperar el parto por las vias naturales, es aconsejable la aplicación de forceps para el desprendimiento y la episiotomía, dada la deficiencia de la pared uterina; así como el uso de la raquianestesia cuando se' presenta distocia de partes blandas por rigidez de ellas. Cuando el útero vacío se convierte en tumor previo, la operación cesárea es de absoluta necesidad y su realización no tendrá, por efecto de la duplicidad orgánica, ninguna particularidad técnica especial. El único punto discutible es el de saber si conviene o no extirpar en seguida el útero previo, si se aplaza esta exéresis para otra ocasión o si se conservan los dos cuerpos uterinos. Si ha de hacerse la hemihisterectomía parece de lógica, por razones de más fácil hemostasis, aplazarla para el final del puerperio y hacerla sub-total. En el caso de nuestra paciente no nos pareció necesaria la extirpación del útero no gestante y posteriormente, en el $2^{\circ}$ y 3 o embarazo, hubimos de encontrar que éstos evolucionaron normalmente en él, hasta la práctica de la segunda y la tercera cesárea.

A continuación presentamos un resumen del caso clínico que motivó la presente monografía.

Instituto Materno_Infantil "Concepción Villaveces de Acosta”. Bogotá. Servicio del profesor Rafael Ramírez Merchán. Historia número 8144. VII-12-54. Nombre: R. D. de T. Edad: treinta años. Ocupación: hogar. Procedencia: Bogotá. Gestante II. Para I.

\section{Antecedentes de importancia:}

Personales: Tifoidea hace 4 años. Ginecológicos: Menarca a los catorce años. Ciclo $30 \times 3$. Dismenorrea. Flujo amarillo cáustico.

Obstétricos: Según el relato que hace la paciente, parece que tuvo un aborto completo de 3 meses, espontáneo.

Al examen clínico general no se encuentra nada especial, aparte de modificaciones y síntomas de los órganos genitales, taıes como calostrorrea y tumor hipogástrico con dolor espontáneo y provocado en la fosa ilíaca derecha.

Pelvimetría externa: B. E. 23 B. C. 28 . B. T. 32 . C. E. 20. 
La paciente acude a la Consulta Prenatal del Instituto el 12 de julio de 1954 y en cuanto a su afección relata que estuvo en amenorrea desde el 18 de diciembre de 1953 hasta el 4 de marzo de 1954, cuando presentó hemorragia vaginal acompañada de la expulsión de un fragmento de tejido al parecer ovular. Después de suspenderse totalmente, la hemorragia repitió el día 12 del mismo mes de marzo con mayor intensidad y entonces expulsó "una bolsa llena de líquido". Desde entonces no ha vuelto a sangrar, pero dice que desde hace 15 días ha experimentado dolor en la fosa ilíaca derecha, que la ha obligado a guardar cama. Las características de la hemorragia relatada por la paciente hacen pensar que se trató de un aborto completo de 3 meses.

Al examen clínico se encuentra una paciente en buenas condiciones generales. Ruidos cardíacos normales con frecuencia de 70 por minuto; tensión arterial: máximo 100. Mínimum 60. Todos los demás órganos son normales, hecha excepción de la existen. cia de un tumor hipogástrico y de dolor espontáneo y provocado eni la fosa ilíaca derecha. Este tumor, redondeado, que alcanza sobre el púbis una altura de 14 centímetros, está desviado hacia la derecha, es poco móvil y la tentativa de su desplazamiento provoca dolor.

Se practica examen ginecológico y se encuentra al tacto vaginal una vagina de nulípara con un tabique sagital completo que la divide en dos cavidades vaginales completamente separadas. En el fondo de la hemivagina derecha se encuentra un cervix blando, corto, cerrado y colocado hacia adelante. En la hemi. vagina izquierda se encuentra otro cuello uterino de las mismas características del derecho pero un poco más duro y colocado hacia atrás. Los hallazgos del tacto vaginal fueron corroborados en el examen con "speculum". En el mismo tacto vaginal combina. do con palpación abdominal, así como en tacto rectal, se puede apreciar la existencia de dos cuerpos uterinos, grávido el derecho y un poco aumentado de tamaño el izquierdo. Diagnóstico: Secundigestante de 4 meses. Utero didelfo. Embarazo localizado en el útero derecho-(Doctor Guillermo Navas Angel).

Se ordena la hospitalización de la paciente y su permanencia en el Servicio hasta el final del embarazo. Se practicaron exámenes de laboratorio rutinarios con los siguientes resultados: $R$. de Galli_Mainini: positiva. R. serológicas: negativas. Hemo_ grama: normal. Examen parcial de orina: normal. El dolor pélvico fue tratado con éxito por medio de enemas laudanizados pa- 
ra retener y con inyecciones intramusculares de Progesterona de absorción lenta que fueron administradas cada 20 días durante todo el embarazo.

Cuando el embarazo evolucionaba en el octavo mes se practicó un estudio radiográfico inyectando la sustancia opaca ( $\mathrm{Li}$ piodol) a través del cuello uterino izquierdo. De esta manera se hace histerosalgografía del útero y la trompa izquierda en contraste con el esqueleto del feto, alojado en el útero derecho. En la imagen radiográfica (Figuras 1 y 2), se aprecia también una deformación de la cavidad uterina izquierda determinada por la presencia de un mioma submucoso.

El trabajo de parto comenzó espontáneamente el 15_XII-54 a las 22 horas. En sucesivos exámenes de control practicados se pudo apreciar falta de encajamiento de la presentación, trabajo regular, borramiento y dilatación progresiva del cuello uterino derecho, más no así del izquierdo que solo sufrió el borramiento. El útero izquierdo, no grávido, se aprecia colocado por delante de la presentación, como tumor previo. La dilatación del cuello derecho llegó a ser de 6 centímetros, la bolsa amniótica se mantuvo intacta y a través de las membranas se diagnosticó presen. tación de vértice ODP, muy deflejada. En estas condiciones se decidió practicar la operación cesárea.

Cirujanos: doctores Hernando Navas Angel y Jaime Ramirez Sánchez. Anestesiólogo: doctor Primitivo Correal Barrios. Fecha: XII-16-54. Anestesia raquídea.

Laparatomia mediana infraumbilical. Cesárea segmentaria transperitoneal con apertura del útero por disociación transversal de las fibras musculares del segmento. Se extrae feto vivo, masculino, de 2.600 gramos de peso e inmediatamente se aplica 1 ampolla de Pitocin i. v. Se extrae la placenta y se sutura la brecha uterina en dos planos musculares, perforante total el primero e invaginante el segundo, usando en ambas catgut cromado No 1. Después de la sutura del peritóneo uterino, se hace revisión y "toilette" de cavidad abdominal, así como exploración de los genitales internos que para tal efecto son exteriorizados y fotografiados (Figura 3). En la exploración se encuentra: útero derecho, sitio de la preñez, en retracción normal post-alumbramiento; útero izquierdo de una dimensión de 8 centímetros en su eje longitudinal, reblandecido y que a la palpación presenta un mioma submucoso. Cada uno de los dos úteros está provisto de un cuerno externo, de una trompa a la que se adhiere un ovario nor- 


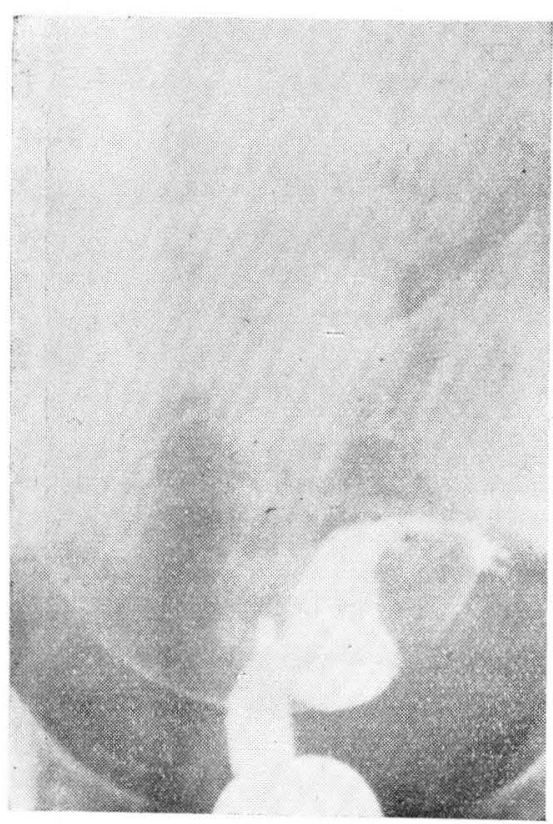

Figura número 1

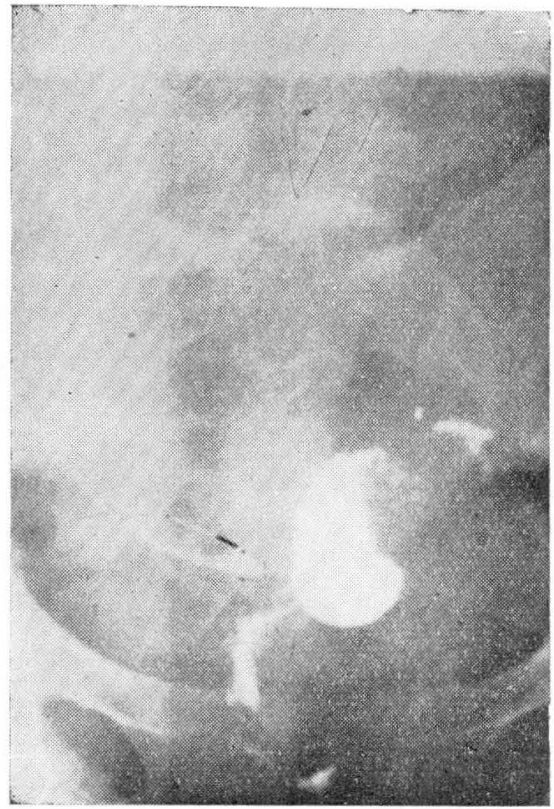

Figura número 2

mal y de un ligamento redondo. Los dos úteros están adheridos entre sí a nivel de los cuellos, pero se comprueba que no existe comunicación entre sus cavidades. Se procede entonces al cierre de la pared abdominal en cinco planos y en la forma acostumbrada. La madre y el niño salen de la sala en buenas condiciones.

Después de un post-operatorio normal, la paciente sale del servicio en buenas condiciones el 27.XII_54, con la recomendación de volver al cabo de dos meses para practicarle histerosalpingografía bilateral, to cual no cumplió.

Reingresa nuevamente la paciente el 4-I-56 con embarazo de ocho mese's y medio, en evolución normal. Esta vez el feto está alojado en el útero izquierdo en presentación de pelvis completa. El 30 del mismo mes inició espontáneamente el trabajo de parto que evolucionó con contracciones regulares, ruptura precoz y espontánea de las membranas y dilatación lenta del cuello izquierdo. El útero derecho evoluciona como tumor previo y por esa razón se decide practicar cesárea, que es ejecutada por los doctores Mario Sanz Araos y Jorge Rengifo Arboleda. Se extrae feto vivo, masculino, de 2.900 gramos de peso y en la exploración 


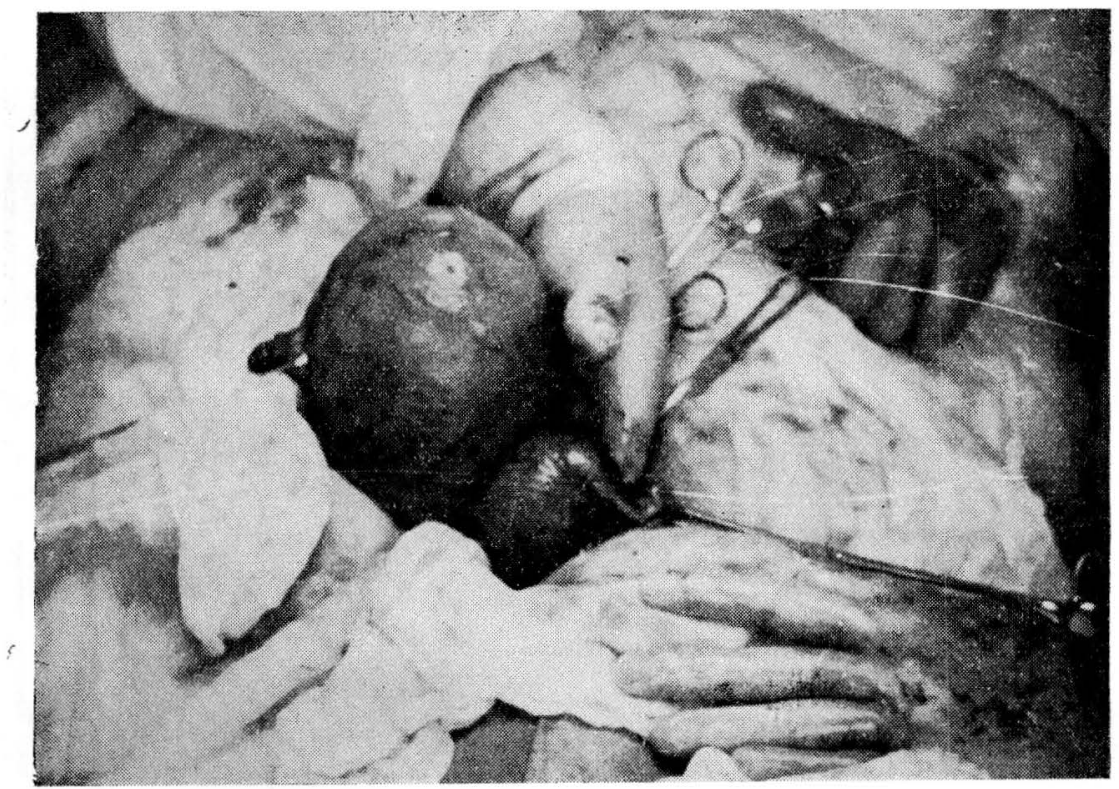

Figura número 3

se aprecia el útero izquierdo bien retraído después de su sutura y el derecho aumentado de tamaño y con algunos núcleos fibromatosos. Post-operatorio normal.

E1 16-VI_57 la paciente acude nuevamente al Servicio y se hospitaliza con embarazo de 8 meses, alojado en el útero izquierdo e iniciando trabajo de parto prematuro. En vista de que a pesar de la medicación indicada para detener el proceso final del embarazo el trabajo sigue su curso, los doctores Miguel Páez y Jaime Barragán practican la tercera operación cesárea, iterativa para el útero izquierdo, obteniendo feto femenino, vivo, de 2.200 gramos de peso que es atendido en el Servicio de Prematu. ros. Después de post_operatorio normal de la madre y de normal aumento de peso de la niña, salen del Instituto en buenas condiciones.

\section{Resumen}

Con motivo de un caso de duplicidad genital femenina, se hace un estudio de Ias malformaciones útero-vaginales congénitas de este género, analizando los aspectos embriológicos, etio. 
patogénicos, de clasificación, de diagnóstico y se hacen consideraciones acerca de los problemas ginecológicos y obstétricos que surgen en las pacientes afectadas por esta clase de anomalías.

Al final se presenta el caso que motivó este estudio haciendo un resumen de la historia clínica.

\title{
BIBLIOGRAFIA
}

1. Encyclopedis Médico-Chirurgicale. Obstetrique. Volume II.

2. Nubiola. Zárate. Tratado de Obstetricia. Tomo II. 1951.

3. De Lee. Groenhill. Principles and Practice of Obstetrics. 8th Edition. 1945.

4. Greenhill. Year Book of Obstetrics \& Gynecology. 1953-1954. Series.

5. R. Keller et A. Ginglinger. La Chirurgie en Obstetrique. 1946.

6. Hernando Echeverri V. Tres casos de anomalías vagino-uterinas. Rev. Col. de Obs. y Gin. Vol. VI No 2. Nov., Dic. 1954.

\section{MEMORIAS DE LA SEGUNDA CONVENCION NACIONAL DE OBSTETRICIA Y GINECOLOGIA}

Una publicación especial de la Sociedad Colombiana de Obstetricia y Ginecología, dedicada al Cuerpo Médico del país.

Contiene todos los trabajos científicos presentados en el II Congreso celebrado en Cali en diciembre de 1955.

\author{
DE VENTA EN: \\ SOCIEDAD COLOMBIANA DE OBSTETRICIA \\ Y GINECOLOGIA
}

Bogotá, D. E. Avenida Jiménez, número 5-16. Oficina 302. Teléfono 432-511. Apartado Nacional 3010.

VALOR DEL EJEMPLAR: \$20,00 MONEDA CORRIENTE. 